\title{
Beam Polarimetery at HERA
}

\author{
W. Lorenzon \\ (on behalf of the HERMES collaboration and the HERA polarimeter group) \\ Randall Laboratory of Physics, University of Michigan, Ann Arbor, MI 48109-1120, USA \\ and \\ Deutsches Elektronen-Synchrotron, Notkestrasse 85, 22603 Hamburg, Germany
}

\begin{abstract}
The polarization of the $27.5 \mathrm{GeV}$ electron/positron beam of the HERA $e p$ collider ring is routinely measured with two independent Compton polarimeters. The transverse Compton polarimeter was developed as a tool for the HERA operators to tune the machine for high beam polarization. Currently it is also used to provide reliable beam polarization measurements for the HERMES experiment which investigates the spin structure of the nucleon. An additional Compton polarimeter, which was commissioned over the last twelve months, provides an independent measurement of the longitudinal beam polarization between the spin rotators at the HERMES experiment. The operation of the two polarimeters is presented and compared to each other.
\end{abstract}

\section{INTRODUCTION}

In high energy storage rings the electron (positron) spin can become transversely polarized through the Sokolov-Ternov effect [1]: the synchrotron radiation process contains a small asymmetric spin-flip amplitude that enhances the polarization state antiparallel (parallel) to the magnetic bending field. The polarization increases in time according to

$$
P(t)=P_{\max }\left(1-e^{-t / \tau}\right),
$$

where $\tau$ is the polarization build-up time and $P_{\max }$ is the asymptotic polarization.

In an ideal flat machine and in the absence of depolarizing effects the maximum polarization theoretically achievable $\left(P_{\mathrm{ST}}\right)$ is 0.924 . In real machines, there are also depolarizing effects which can counteract the Sokolov-Ternov effect. The strength of these depolarizing mechanisms can be quantified with the time constant $\tau_{D}$. These mechanisms compete with the Sokolov-Ternov build-up, and the value of the asymptotic polarization $P_{\max }$ is determined by the relative strengths of the two processes according to 


$$
P_{\max }=P_{\mathrm{ST}} \frac{\tau_{\mathrm{ST}}}{\frac{1}{\tau_{\mathrm{ST}}}+\frac{1}{\tau_{\mathrm{D}}}}
$$

The effective build-up time $\tau$ is also reduced to

$$
\frac{1}{\tau}=\frac{1}{\tau_{\mathrm{ST}}}+\frac{1}{\tau_{\mathrm{D}}}
$$

The Sokolov-Ternov time constant, $\tau_{\mathrm{ST}}$, for HERA at $27.5 \mathrm{GeV}$ is $37 \mathrm{~min}$. The actual build-up time scales with $P_{\max }$ and will thus be shorter than $\tau_{\mathrm{ST}}$. This can be seen by rearranging Eq. 2 and 3, which gives

$$
P_{\max }=\tau\left(\frac{P_{\mathrm{ST}}}{\tau_{\mathrm{ST}}}\right)
$$

This important feature can be exploited to obtain an independent determination of $P_{\max }$ and thus a scale calibration of the polarization measurement from the actually observed build-up time. For example, $\tau=20 \mathrm{~min}$ would correspond to $P_{\max }=0.50$. Therefore, assuming that the Sokolov-Ternov calculation of the build-up time is exact, the polarization scale can be calibrated using the characteristic rise-time behavior.

For a non-flat machine (i.e. a machine with spin rotators), however, Eq. 2 is not exact. There is an extra factor $(1+\delta)$ in the numerator. This can be seen by inspecting the Derbenev-Kondratenko formula [2] which generalizes the Sokolov-Ternov formula to take depolarizing effects into account. When the spin rotators are off, $\delta$ is so small that it can be neglected. With the spin rotators on, $\delta$ cannot be estimated reliably but must be determined experimentally.

\section{HERA Spin Rotators}

The HERMES experiment, located in the East section of the HERA ep collider ring, requires longitudinal beam polarization. This is accomplished with two spin rotators [3] located at the entrance and exit of the East straight section, that precess the spin direction from vertical to longitudinal at the HERMES target position. Currently, only HERMES uses spin rotators. The spin rotators for the $\mathrm{Hl}$ and ZEUS collider experiments will be installed in the $1999 / 2000$ shutdown period.

The transverse beam polarization is measured in the HERA West section whereas the longitudinal beam polarization is measured near the HERMES target in the HERA East section. The degree of polarization is invariant over the entire ring; thus the two Compton polarimeters must measure the same polarization. A prominent feature of the HERA spin rotators is that they can be arranged to reverse the longitudinal spin direction [3]. This is an important tool for any experiment at HERA that takes data with polarized beams to reduce systematic uncertainties in the measured physics observables. 


\section{POLARIZATION MEASUREMENT}

The method for measuring the beam polarization with either polarimeter installed in the HERA storage ring is based on the Compton scattering process. Here, we summarize briefly Compton scattering of circularly polarized laser light on a polarized electron beam, and describe how the transverse and longitudinal polarization of the beam is measured. The transverse polarimeter has been described in great detail in the past $[4,5]$; therefore, more emphasis will be put on the new longitudinal polarimeter.

\section{Compton Scattering}

Compton polarimeters utilize the spin-dependent cross section for Compton scattering of polarized photons on electrons. The differential Compton cross section can be written as a function of the initial electron and photon polarizations $\mathbf{P}$ and $\mathbf{S}$. Since only vertical and longitudinal polarization components of the electron beam are measured, averaging over the horizontal component $x$ leads to [4]

$$
\frac{d \sigma_{c}}{d \Omega}(\mathbf{S}, \mathbf{P})=\frac{1}{2} r_{0}^{2}\left(\frac{k_{f}}{k_{i}}\right)^{2}\left[\Sigma_{0}(\theta)+S_{1} \Sigma_{1}(\theta, \phi)+S_{3}\left\{P_{\mathrm{Y}} \Sigma_{2 \mathrm{Y}}(\theta, \phi)+P_{\mathrm{Z}} \Sigma_{2 \mathrm{Z}}(\theta)\right\}\right]
$$

with $S_{1}$ and $S_{3}$ the linear and circular components of the initial photon polarization, $P_{\mathrm{Y}}$ and $P_{\mathrm{Z}}$ the vertical and longitudinal components of the initial electron polarizations, $r_{0}$ the classical electron radius, and $k_{i}, k_{f}$ the initial and final photon momenta in the electron rest frame. $\Sigma_{0}$ contributes to the unpolarized cross section, $\Sigma_{1}$ and $\Sigma_{2 Y}$ depend on the azimuthal scattering angle $\phi$ and are used for the vertical polarization measurement, and $\Sigma_{2 Z}$ is used for the longitudinal polarization measurement.

\section{Measurement of Transverse Polarization}

The transverse polarization $\left(P_{\mathrm{Y}}\right)$ is measured in a position sensitive calorimeter using the up-down asymmetries in the backscattered Compton photons. The "single-photon method" is used where the laser intensity is chosen such that the probability for multiple Compton photons per bunch crossing is about $1 \%$ and the energy and position of each single photon is used for the analysis. This is achieved with a $10 \mathrm{~W}$ continuous beam of $2.4 \mathrm{eV}$ photons from an argon-ion laser. The laser beam is transported through remotely controlled mirrors and lenses over about $200 \mathrm{~m}$ to the $e \gamma$ interaction point in the HERA tunnel. The photon polarization is switched with a frequency of $84 \mathrm{~Hz}$ 
by a Pockels cell. The backscattered Compton photons are detected in a tungsten/scintillator sandwich calorimeter about $65 \mathrm{~m}$ from the interaction point. The electron beam optics at the interaction point is such that the Compton photons converge to a vertical focus near the location of the calorimeter. This choice, together with the very small electron beam emittance, make possible the measurement of the Compton photon angle to a precision of a few $\mu \mathrm{rad}$. The calorimeter is split into an optically decoupled upper and lower part. The energy of the incoming photon is the sum of the energies of the two halves, $E_{\gamma}=E_{u}+E_{d}$, and the vertical distance of the Compton photons to the center of the calorimeter is measured using the asymmetry of the energies $\eta(y)=\left(E_{u}-E_{d}\right) /\left(E_{u}+E_{d}\right)[4]$.

There are two ways to measure the vertical polarization [4]:

1. The vertical polarization can be obtained from measurements of the asymmetry

$$
\mathcal{A}\left(y, E_{\gamma}\right)=\Delta S_{1} \Sigma_{1}+\Delta S_{3} P_{\mathrm{Y}} \Sigma_{2 Y}
$$

with e.g. $\Delta S_{1}=\frac{1}{2}\left(S_{1, L}-S_{1, R}\right)$, where $S_{1, L}$ and $S_{1, R}$ are the degrees of linear polarization of the laser light. Note that the asymmetry measurement not only depends on the circular, but also the linear component of the laser light.

2. The polarization $P_{\mathrm{Y}}$ can also be obtained from the shift of the mean vertical positions $\langle y\rangle$ measured with left and right circularly polarized light

$$
\Delta\langle y\rangle\left(E_{\gamma}\right)=\frac{1}{2}\left(\langle y\rangle_{\mathrm{L}}-\langle y\rangle_{\mathrm{R}}\right)=P_{\mathrm{Y}} \Delta S_{3} \Pi\left(E_{\gamma}\right)
$$

The analyzing power $\Pi\left(E_{\gamma}\right) \leq 170 \mu \mathrm{m}$ is equal to the shift measured when $\triangle S_{3} P_{\mathrm{Y}}=1$. Tis is the standard method used to measure $P_{\mathrm{Y}}$.

The statistical error in the transverse polarization $\delta P_{Y}$ obtained in one minute is about 0.01 to 0.02 depending whether the data is taken in the beginning or towards the end of a fill. The beam currents can vary between 40 to $10 \mathrm{~mA}$ during a fill.

\section{Measurement of Longitudinal Polarization}

In contrast to the small spatial asymmetry measured with the transverse calorimeter, the measurement of the longitudinal polarization $\left(P_{\mathrm{Z}}\right)$ is based on large asymmetries in the energy distributions of the backscattered photons, with $\mathcal{A}\left(E_{\gamma}\right)=\Delta S_{3} P_{\mathrm{Z}} \Sigma_{2 \mathrm{Z}}$. 
The energy-dependent analyzing power $\Sigma_{2 Z}$ is shown in Fig. 1. The longitudinal polarization $P_{\mathrm{Z}}$ can be obtained from the measurement of the energydependent asymmetry under reversal of the laser photon helicity using the "single-photon method". However, because the HERMES gas target produces intense bremsstrahlung background at the calorimeter of the longitudinal polarimeter, the "multi-photon method" is preferred. In contrast to the "singlephoton method" utilized in the transverse polarimeter, where the energy of every individual Compton photon is analyzed, the "multi-photon method" is based on a measurement of the total energy deposited in the detector by about 1000 Compton photons per bunch crossing. This is achieved with a frequency doubled, pulsed YAG laser. It produces $2.3 \mathrm{eV}$ photons with a repetition rate between $0.1-100 \mathrm{~Hz}$ and pulse energies from 1 to $250 \mathrm{~mJ}$. The pulsed laser beam is guided with remotely controlled mirrors and lenses in a $72 \mathrm{~m}$ long stainless steel vacuum pipe to the $e \gamma$ interaction point. The laser is pulsed at $100 \mathrm{~Hz}$ and a Pockels cell switches the photon polarization before each laser pulse.

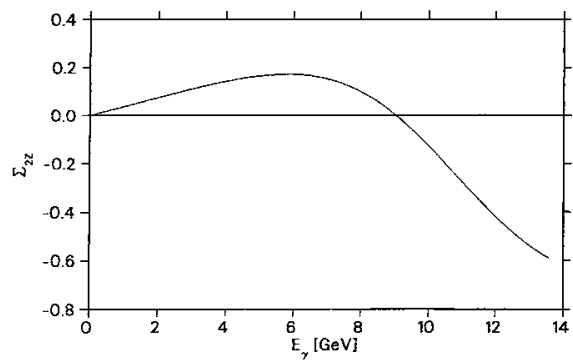

FIGURE 1. The energy-dependent analyzing power $\Sigma_{2 Z}$ plotted versus the photon energy $E_{\gamma}$ for a $2.3 \mathrm{eV}$ photon scattered off a $27.5 \mathrm{GeV}$ electron. At the Compton edge, at $13.6 \mathrm{GeV}$, the asymmetry is close to -0.60 .

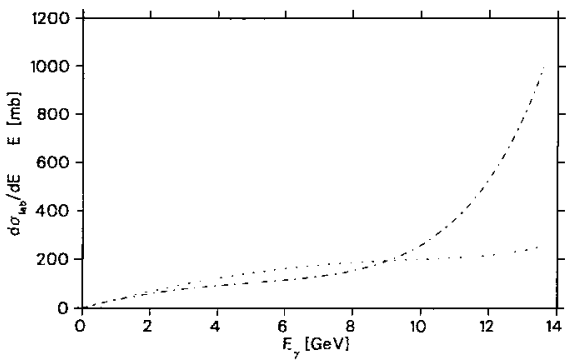

FIGURE 2. Energy weighted spectra of the scattered photons in the lab frame for scattering with longitudinally polarized electrons for the cases of $S_{3} P_{Z}=+1$ (dotted curve) and $S_{3} P_{\mathrm{Z}}=-1$ (dash-dotted curve).

Due to the enormous kinematic boost from the beam (Lorentz factor is $E / m_{e} \approx 54,000$ ), most backscattered photons are contained in a narrow cone centered along the initial electron beam direction. They are detected in a calorimeter $54 \mathrm{~m}$ from the interaction point. It consists of four optically decoupled, radiation hard $\mathrm{NaBi}\left(\mathrm{WO}_{4}\right)$ Cerenkov crystals, arranged in a $2 \times 2$ array. The energy of the incoming photons is the sum of the energies of all four crystals, and the $2 \times 2$ configuration allows a precise determination of their horizontal and vertical positions. The spatial distribution of the incoming photons are mainly determined by the beam optics; therefore, at the calorimeter position, the cone has a radius of $7 \mathrm{~mm}$ for photon energies above $500 \mathrm{MeV}$. Compton photons below that energy threshold contribute less than $0.5 \%$ to the asymmetry and can be neglected. Therefore, in the "multi-photon 
method" the longitudinal polarization is obtained from measurements of the energy weighted asymmetry

$$
\mathcal{A}\left(\Sigma E_{\gamma}\right)=\triangle S_{3} P_{\mathrm{Z}} \Sigma_{\mathrm{Z}_{1 \mathrm{r}}}
$$

with

$$
\Sigma_{Z_{l \mathrm{r}}}=\frac{\Sigma_{l}-\Sigma_{r}}{\Sigma_{l}+\Sigma_{r}}, \quad \text { and } \quad \Sigma_{i}=\int_{E_{\min }}^{E_{\max }}\left(\frac{\mathrm{d} \sigma}{\mathrm{dE}}\right)_{i} \cdot \mathrm{E} \cdot \mathrm{dE}, \quad \text { where } i=l, r \text {. }
$$

The energy weighted analyzing power $\Sigma_{\mathrm{Z}_{\mathrm{lr}}}$ is 0.184 . The energy weighted spectra with longitudinal electron polarization $P_{\mathrm{Z}}=1$ and photon polarization $S_{3}= \pm 1$ are shown in Fig. 2. Assuming 1000 backscattered Compton photons are produced per laser pulse, $6820 \mathrm{GeV}$ is deposited in the calorimeter for an unpolarized electron beam, since the average energy deposited per Compton photon is $6.8 \mathrm{GeV}$. For the two polarization states, one measures two peaks separated by $1770 \mathrm{GeV}$ for a 0.70 polarized electron beam, as shown in Fig. 3. (In comparison, for the transverse polarimeter, a spatial shift of $100 \mu$ has to be detected). The measured energy peak positions depend on the luminosity, and the electron and laser polarizations, $P_{Z}$ and $S_{3}$.
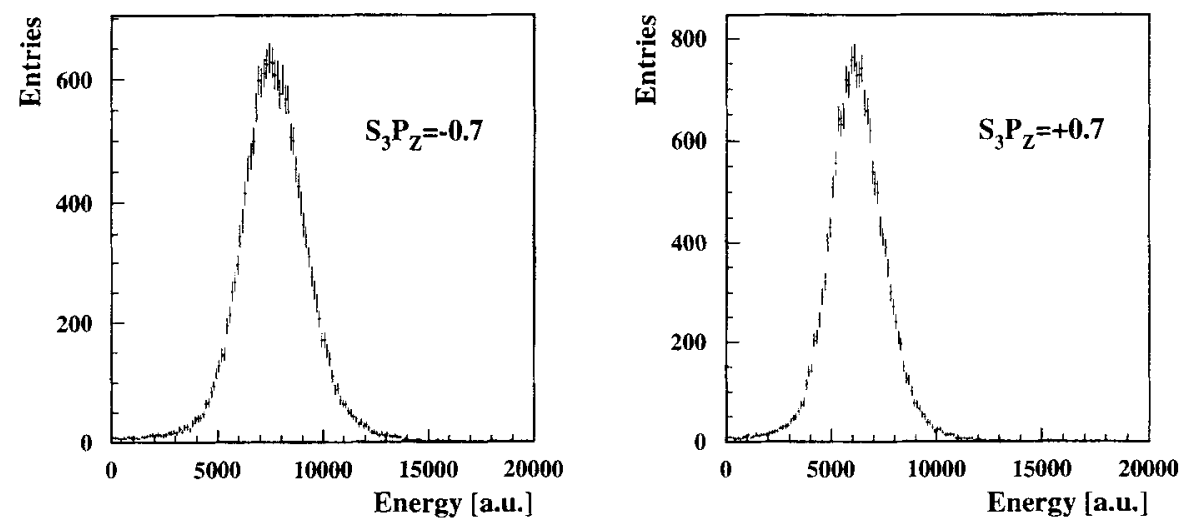

FIGURE 3. Histogram for 11,000 events collected in "multi-photon mode". The polarization is determined from the centroid difference of these two peaks, with an extracted value of $70 \%$. The two peaks are the results of changing the laser helicity.

The polarization is determined from the centroid difference of these two peaks divided by the energy weighted analyzing power (0.184) under the assumption that $\Delta S_{3}=1$. Note that it is not necessary to know the absolute energy scale, it is sufficient to know that the energy scale is linear. This can be checked by varying the laser power. 
To achieve a statistical error in $\delta P_{Z}$ of 0.01 , a measurement takes about one minute. The laser power can be adjusted easily to keep the luminosity constant. The background is measured between each laser pulse and is negligible in the "multi-photon method".

\section{ABSOLUTE POLARIZATION SCALE}

Within a factor of two, both Compton polarimeters deliver statistical errors in the polarization of about 0.01 per minute. The main goal for building the newly commissioned longitudinal polarimeter was to have a second, independent measurement of the beam polarization, and to reduce the overall systematic uncertainty in the absolute polarization value. Experience has shown, each polarimeter is available for reliable polarization measurement about $90 \%$ of the HERMES data production time. The overall efficiency to deliver valid polarization values to HERMES is close to $100 \%$.

As discussed in Section II.B and II.C, the polarization of the HERA beam can be determined from basic principles by starting from the spin-dependent Compton cross section. This requires a very detailed understanding of all the components involved as well as an accurate determination of the analyzing power, $\Pi\left(E_{\gamma}\right)$, for the transverse polarimeter, or the energy weighted analyzing power, $\Sigma_{\mathrm{Z}_{\mathrm{l}}}$, for the longitudinal polarimeter, which are determined from Monte Carlo simulations.

A much more straightforward way of obtaining the absolute polarization scale is by using the rise-time calibration described in Section I.

\section{Rise-Time Calibration}

In 1994, rise-time curves were taken to get an absolute calibration of the transverse beam polarization. This was done with the spin rotators deactivated, i.e. with a flat machine. Analysis of those eleven rise-time curves which passed quality cuts indicated that the polarization scale predicted by Monte Carlo simulations resulted in values that were $6 \%$ too high. The systematic uncertainty associated with that scale calibration was $3.2 \%$, a considerable improvement from the $9 \%$ systematic error assigned to the Monte Carlo simulations [4].

In 1997, after completion of the longitudinal polarimeter, another set of rise-time calibration runs were taken to compare the performance of the longitudinal and transverse polarimeters, and to study the effect of the spin rotators on the absolute polarization scale. One of these rise time curves, taken simultaneously with the transverse and longitudinal polarimeters, is shown in Fig. 4. There, the polarized beam was depolarized about six hours into the fill by activating a kicker magnet at a depolarizing resonance frequency. After about ten minutes this depolarizer was turned off and the polarization 
started to rise again. At the end of the rise-time curve the electron beam was depolarized again to check for stability in the beam conditions. In case of unstable machine conditions, the depolarizing frequency would have drifted. This was not the case for the curves shown. The agreement in the fitted polarization build-up time $\tau$ for both polarimeters is better than $1 \%$ which is not surprising since the polarization is expected to be invariant over the entire ring. Using $\tau$, both polarimeters can be calibrated absolutely up to the factor $(1+\delta)$ discussed in Section I. The scale correction needed for the longitudinal polarimeter, which was already applied in Fig. 4, lowered the measured values by $20 \%$. It is believed that this large number can be reduced in the near future after more detailed Monte Carlo studies and more extensive systematic studies are completed.
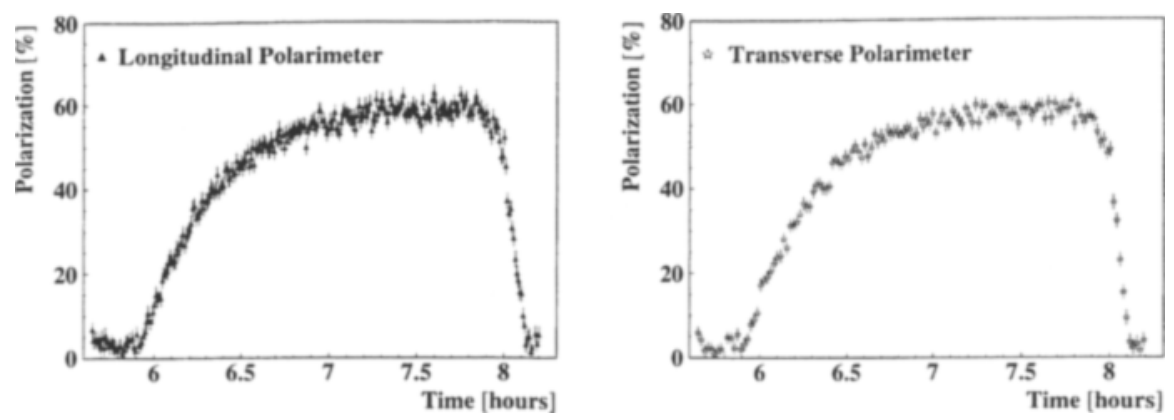

FIGURE 4. Rise-time curve taken simultaneously with the longitudinal and transverse polarimeters to compare build-up time and general performance of both polarimeters. At the beginning and the end of the spectrum, the beam was depolarized on purpose.

In 1997, the machine conditions were much more stable than in 1994. Therefore, new rise-time calibration data were taken successfully which yielded a factor $1.01 \pm 0.01$ as compared to the calibration taken in 1994. In order to improve the overall polarization scale uncertainty, another set of measurements has to be taken, again with the spin rotators turned off, therefore re-establishing a flat machine. If a similar accuracy can be obtained, then the absolute polarization scale is known to better than $2 \%$ and the unknown correction $\delta$ is known to the same precision. Current plans forsee that those measurements will take place in late fall 1997.

\section{Polarization Uncertainties}

The overall error in $\Delta \mathrm{P} / \mathrm{P}$ for the transverse polarimeter is shown in Table 1. The independent statistical and systematic errors are added in quadrature, whereas the scale uncertainty obtained from the rise-time calibration is added 
linearly. In order to reduce the overall error in the polarization measurement to the $3 \%$ level, rise-time studies with flat spin rotators have to be performed.

TABLE 1. Fractional errors in $\triangle \mathrm{P} / \mathrm{P}$ for transverse polarimeter.

\begin{tabular}{rccc} 
& 1995 & 1996 & 1997 expected \\
\hline Statistical (10 min.) & $0.8 \%$ & $0.5 \%$ & $0.5 \%$ \\
Light polarization & $0.5 \%$ & $0.5 \%$ & $0.5 \%$ \\
Calorimeter Calibration & $2.0 \%$ & $0.5 \%$ & $0.5 \%$ \\
\hline Quadratic Sum & $2.2 \%$ & $0.9 \%$ & $0.9 \%$ \\
Rise-time correction & $3.2 \%$ & $3.2 \%$ & $\sim 2 \%$ \\
\hline Typical Uncertainty (linear Sum) & $5.4 \%$ & $4.1 \%$ & $\sim 3 \%$ \\
\hline
\end{tabular}

\section{OUTLOOK}

There is a very interesting feature of the longitudinal polarimeter. Due to the high backscattered photon rates $\left(10^{3}\right)$ and the high repetition rate of the YAG laser $(100 \mathrm{~Hz})$, the polarization of individual bunches can be measured to $2 \%$ statistical precision in approximately twenty seconds. Thus in about ten minutes, the polarization of each bunch is known to $5 \%$, as shown in Fig. 5. This enables us to study beam-beam interaction and beam orbit effects on the polarization. Not all the electron/positron bunches collide with proton bunches. Those so-called pilot bunches which do not collide can have significantly different polarization values than the bunches that do collide with protons. This has been very valuable information for the machine physicists.

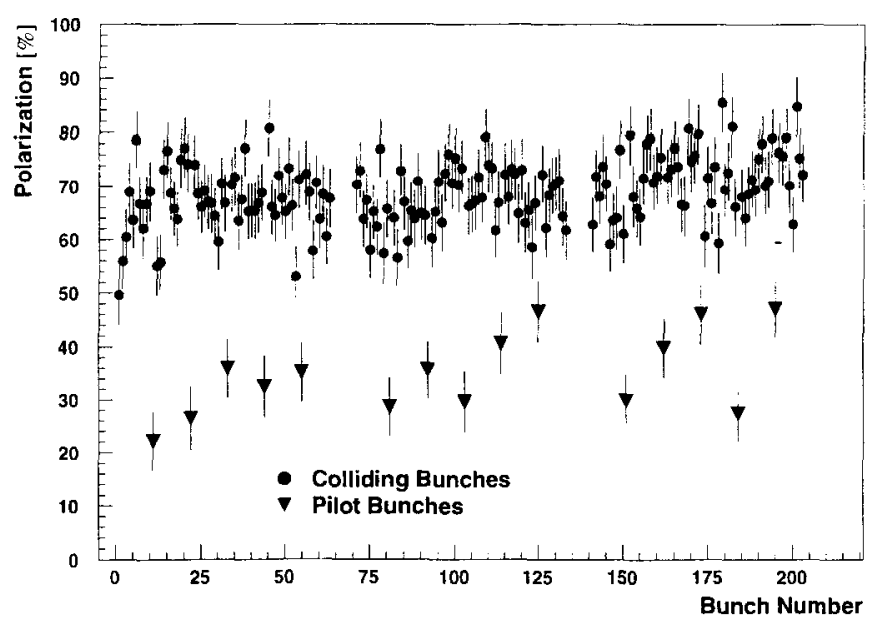

FIGURE 5. Single bunch polarization values for colliding and non-colliding (pilot) bunches are shown. 
One possible explanation of this effect is that in fact colliding bunches have different tunes, and therefore different orbit corrections than pilot bunches due to beam-beam interactions. During polarization tuning by the machine operators the average beam polarization is optimized. This means that the polarization for colliding bunches is preferentially optimized, since the ratio of pilot to colliding bunches is about 12:1.

The ability to measure the polarization of individual bunches can also be used to better monitor the stability of the positron beam in conjunction with the concept of partial depolarization of the beam. In this concept only a fraction of the 189 filled bunches are depolarized, after all the bunches have reached asymptotic polarization values. Rise-time measurements can then be performed on these few depolarized bunches, while the rest of the bunches stay at constant levels. This scheme allows us to monitor the stability of the positron beam during rise-time curves, because any instability could be observed as a non-statistical change of the asymptotic polarization values. Stable beam conditions are essential to extract meaningful rise-time curves. More importantly, it also allows us to check the calibration of the polarization scale continuously by sacrificing e.g. one single bunch which can be depolarized every few hours.

\section{ACKNOWLEDGEMENTS}

I wish to thank my colleagues in the HERMES collaboration and the HERA polarimeter group. I acknowledge P. Schüler, H. Fischer and C.A. Miller for critical reading of the manuscript. The author's research is supported in part by the U.S. National Science Foundation, Nuclear Physics Division under grant No. PHY-9724838. I thank the University of Michigan and DESY for support while on research leave.

\section{REFERENCES}

1. A.A. Sokolov and I.M. Ternov, Sov. Phys. Doklady 8 (1964) 1203.

2. Y.S. Derbenev and A.M. Kondratenko, Sov. Phys. JETP 37, $\mathrm{N}^{0} 6$ (1973) 968.

3. J. Buon and K. Steffen, Nucl. Instr. Meth. A256 (1986) 248.

4. D.P. Barber et al., Nucl. Instr. Meth. A329 (1993) 79.

5. D.P. Barber et al., Nucl. Instr. Meth. A338 (1994) 166. 